\title{
THE NUMERICAL SIMULATION OF GREEN WATER LOADING INCLUDING VESSEL MOTIONS AND THE INCOMING WAVE FIELD
}

\author{
K.M.Theresa Kleefsman, G.Erwin Loots, \\ Arthur E.P. Veldman \\ Inst. of Mathematics and Comp. Science \\ University of Groningen \\ PO Box 800 \\ 9700 AV Groningen \\ The Netherlands \\ \{theresa,erwin,veldman\}@math.rug.nl
}

\author{
Bas Buchner, Tim Bunnik \\ MARIN \\ PO Box 28 \\ 6700 AA Wageningen \\ The Netherlands \\ \{b.buchner,t.bunnik\}@marin.nl
}

\author{
Erik Falkenberg \\ FORCE Technoloy Norway AS \\ Claude Monets Allé 5 \\ 1338 Sandvika \\ Norway \\ ef@forcetechnology.no
}

\begin{abstract}
This paper presents results from simulation of green water loading including vessel motions. The simulation is performed through a domain decomposition: the far field and ship motions are calculated by potential theory and are used to simulate the local flow around the deck of an offshore floater using a NavierStokes solver. In the solver the free surface is displaced using a Volume of Fluid based method, improved by introducing a local height function. First, simulations of an irregular wave, where the velocities at the boundaries of the domain are prescribed using results of a linear diffraction code are performed in order to check wave propagation properties. Then, the same code is used to initiate the simulation of an FPSO in high waves, resulting in green water on the deck.
\end{abstract}

\section{INTRODUCTION}

There is a need for calculation methods for local flow phenomena of wave impact loading and loading from green water on the deck of offshore floaters. There exist good (nonlinear) methods to predict the wave field around the vessel and the vessel motions, but when waves are getting steep and are overturning or flowing over the structure, other methods should be used to calculate the flow and resulting loads.

Green water loading is a highly complex and nonlinear process. In Buchner [2] it was shown that numerical prediction methods for the prediction of green water loading need to take into account the following phases in this process, see Figure 1:

A. Nonlinear swell-up around the bow.

B. 'Dam breaking'-type flow onto the deck.

C. 'Hydraulic jump'-type shallow water flow on the moving deck, focussing into a high velocity water 'jet' when the water fronts from the sides meet.

D. Water impact and water run-up in front of the structure, eventually turning over.

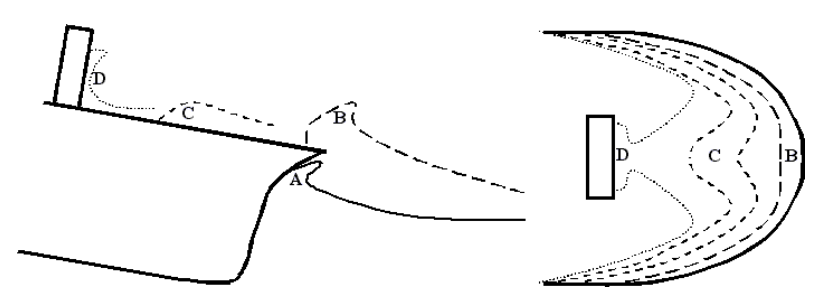

Figure 1. The main phases of the green water problem schematically in side view (left) and top view (right): from the nonlinear relative wave motions in front of the bow, via the complex flow onto and on the deck to the impact on deck structures.

To be able to describe these phenomena, the numerical 
method should be able to deal with complex nonlinear flows. If the focus of the investigation is limited to the local flow around the bow, specifically it should be able to handle:

1. Water entry of a flared bow structure.

2. Complex flow onto the deck, including the discontinuity at the deck edge.

3. 'Hydraulic jump'-type shallow water flow on a moving ship deck.

4. Meeting water flows on the deck.

5. Short duration water impact on a structure.

6. Overturning flow after run-up of the water in front of the structure.

Initial numerical investigations on green water loading focussed on the shallow water flow on the deck, using Glimm's method, see for instance Mizoguchi [12,13], Zhou et al. [16], and Stansberg et al. [15]. With this type of methods only the 'hydraulic jump'-type shallow water flow on the moving deck can be simulated (Phase C). The computational domain can consequently be limited to the area on the deck. The freeboard exceedance around the deck and the related velocities were used as boundary conditions.

In Fekken et al. [4] this approach was extended to the water impact and water run-up in front of the structure (Phase D). Using an improved Volume of Fluid (iVOF) method they were able to simulated the flow on the deck and resulting impact accurately. However, still the computational domain was limited to the area on the deck (see Figure 2). The (measured) freeboard exceedance around the deck was used as boundary condition. Further the deck was not moving in this approach.

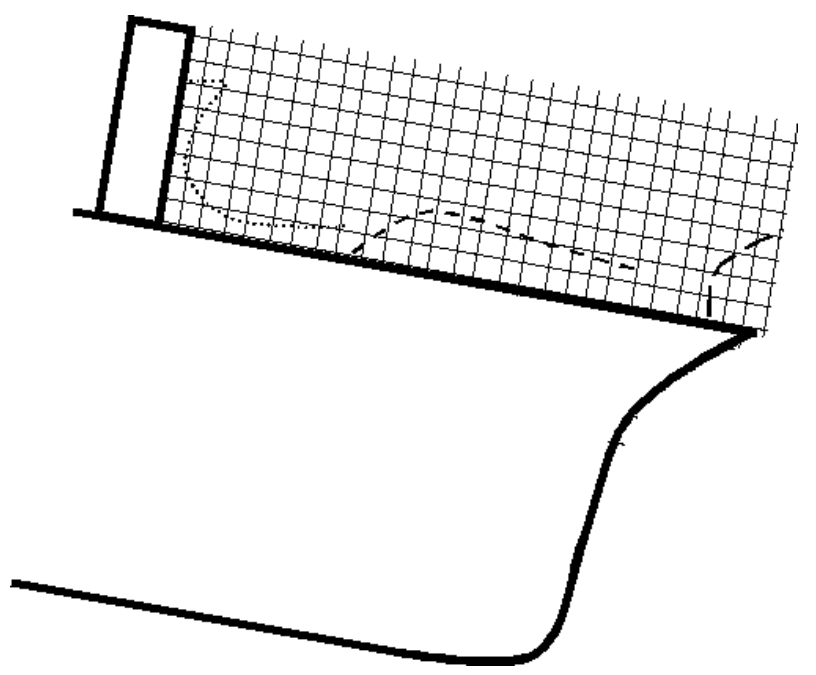

Figure 2. The approach of Fekken et al. [4] where water impact and water run-up are also taken into account
Based on the work done in the SAFE-FLOW project, the present paper makes one step further in this development. The computational domain of the iVOF method is extended to the area outside the bow (see Figure 3), so that nonlinear waves and wave run up can be taken into account in the numerical simulation. As boundary conditions (waves and resulting ship motions), the input of linear diffraction analysis is used. This domain decomposition allows detailed flow simulations in areas with complex nonlinear flows and still limits the computation times. The linear diffraction analysis also provides improved boundary conditions, inflow as well as outflow, for the computational domain.

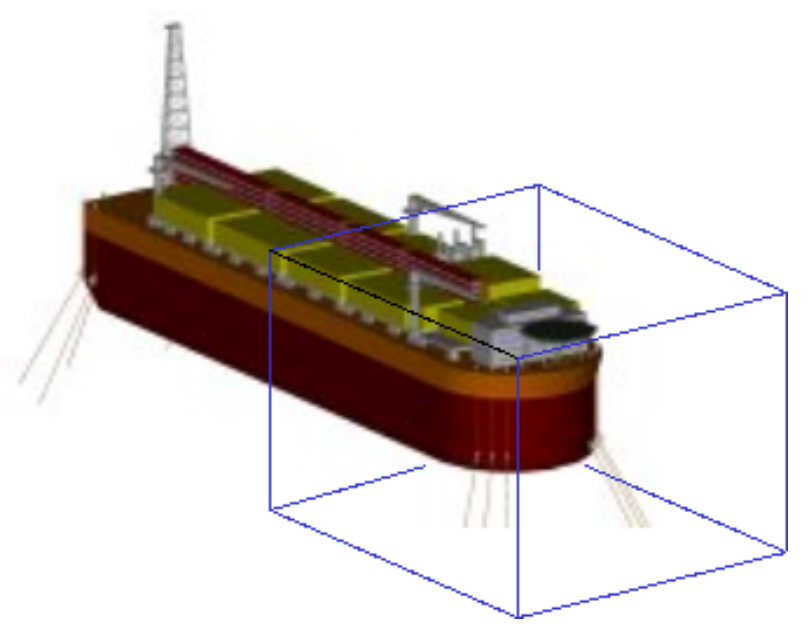

Figure 3. The far field wave kinematics and vessel motions are calculated using a diffraction code and used to initialise the iVOF method for the close surroundings of the vessel's bow.

The iVOF method is incorporated in the program CoMFLOW. It is based on the Navier-Stokes equations for an incompressible, viscous fluid. The equations are discretised using the finite volume method. The displacement of the free surface is done using the Volume of Fluid method first introduced by Hirt and Nichols [8]. To avoid flotsam and jetsam, small droplets disconnecting from the free surface, the VOF-method is combined with a local height function.

COMFLOW has already been used for a number of applications, like sloshing on board tumbling spacecraft [6] and blood flow through arteries [11]. Topics from the maritime industry where COMFLOW has been used are sloshing in anti-roll tanks [3], simulation of dambreak flows as a model for green water flow on the deck, and falling objects in calm water [9].

In this paper results are presented of the simulation of green water on the deck of a floating FPSO, where the domain decomposition method is used as described above. A first attempt for 
this coupling between an outer domain (diffraction code) and an inner domain (COMFLOW) is described. First, the mathematics and numerics used in COMFLOW are described. Then the interface between a diffraction code and the COMFLOW simulation is described. As a first test case, an irregular wave without object in the flow has been run to validate the wave propagation characteristics in COMFLOW. Finally, a green water simulation has been performed, where the results of the COMFLOW simulation can be compared with experiments performed at the Maritime Research Institute Netherlands (MARIN).

\section{GOVERNING EQUATIONS COMFLOW}

Flow of a homogeneous, incompressible, viscous fluid is described by the continuity equation and the Navier-Stokes equations. The continuity equation describes conservation of mass and the Navier-Stokes equations describe conservation of momentum. In conservative form, they are given by

$$
\begin{gathered}
\oint_{\partial V} u \cdot n d S=0 \\
\int_{V} \frac{\partial u}{\partial t} d V+\oint_{\partial V} u u^{\mathrm{T}} \cdot n d S= \\
-\frac{1}{\rho} \oint_{\partial V}(p n-\mu \nabla u \cdot n) d S+\int_{V} F d V .
\end{gathered}
$$

Here, $\partial V$ is the boundary of volume $V, u=(u, v, w)$ is the velocity vector in the three coordinate directions, $n$ is the normal of volume $V, \rho$ denotes the density, $p$ is the pressure, $\nabla$ is the gradient operator. Further $\mu$ denotes the dynamic viscosity and $F=\left(F_{x}, F_{y}, F_{z}\right)$ is an external body force, for example gravity.

\section{Boundary conditions}

At the solid walls of the computational domain and at the objects inside the domain, a no-slip boundary condition is used. This condition is described by $u=0$ for fixed boundaries, and $u=u_{b}$ for moving objects with $u_{b}$ the object velocity.

Some of the domain boundaries may let fluid flow in or out of the domain. Especially, when performing wave simulations, an inflow boundary is needed where the incoming wave is prescribed and at the opposite boundary a non-reflecting outflow condition should be used.

When using the domain decomposition, the velocities at the boundaries of the COMFLOW domain are prescribed using the wave kinematics calculated by the far field solver.

\section{Free surface}

If the position of the free surface is given by $s(x, t)=0$, the displacement of the free surface is described using the following equation

$$
\frac{D s}{D t}=\frac{\partial s}{\partial t}+(u \cdot \nabla) s=0
$$

At the free surface, boundary conditions are necessary for the pressure and the velocities. Continuity of normal and tangential stresses leads to the equations

$$
-p+2 \mu \frac{\partial u_{n}}{\partial n}=-p_{0}+2 \gamma H
$$

$$
\mu\left(\frac{\partial u_{n}}{\partial t}+\frac{\partial u_{t}}{\partial n}\right)=0
$$

Here, $u_{n}$ is the normal component of the velocity, $p_{0}$ is the atmospheric pressure, $\gamma$ is the surface tension and $2 \mathrm{H}$ denotes the total curvature.

\section{NUMERICAL MODEL IN COMFLOW}

To solve the Navier-Stokes equations numerically, the computational domain is covered with a fixed Cartesian grid. The variables are staggered, which means that the velocities are defined at cell faces, whereas the pressure is defined in cell centers.

The body geometry is piecewise linear and cuts through the fixed rectangular grid. Volume apertures $\left(F^{b}\right)$ and edge apertures $\left(A^{x}, A^{y}\right.$, and $\left.A^{z}\right)$ are used to indicate for each cell which part of the cell and cell face respectively is open for fluid and which part is blocked by solid geometry. To track the free surface, the volumeof-fluid function $F^{s}$ is used, which is 0 if no fluid is present in the cell, 1 if the cell is completely filled with fluid and between 0 and 1 if the cell is partly filled with fluid.

The Navier-Stokes equations are solved in every cell containing fluid. Cell labeling is introduced to distinguish between cells of different characters. First the cells which are completely blocked by geometry are called B(oundary) cells. These cells have volume aperture $F^{b}=0$. Then the cells which are empty, but have the possibility of letting fluid flow in are labeled E(mpty). The adjacent cells, containing fluid, are $\mathrm{S}$ (urface) cells. The remaining cells are labeled as F(luid) cells. Note that these cells do not have to be completely filled with fluid. In Figure 4 an example of the labeling is given. 


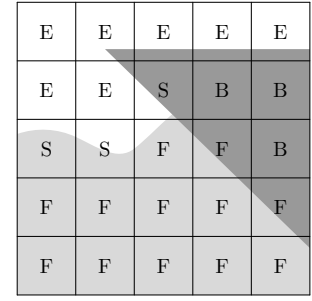

Figure 4. Cell labeling: dark grey denotes solid body, light grey is liquid.

\section{Discretisation of the continuity equation}

The continuity and Navier-Stokes equations are discretised using the finite volume method. The natural form of the equations when using the finite volume method is the conservative formulation as given in Eq. (1) and (2). In this paper, the discretisation is explained in two dimensions. In most situations, this can be extended to three dimensions in a straightforward manner. In

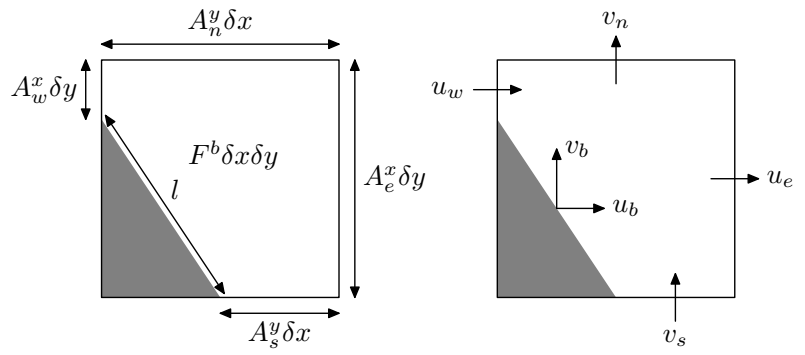

Figure 5. Conservation cell for the continuity equation.

Figure 5 a computational cell is shown, which is cut by the body geometry. When applying conservation of mass in this cell, the discretisation results in

$$
\begin{array}{r}
u_{e} A_{e}^{x} \delta y+v_{n} A_{n}^{y} \delta x-u_{w} A_{w}^{x} \delta y-v_{s} A_{s}^{y} \delta x+ \\
u_{b}\left(A_{e}^{x}-A_{w}^{x}\right) \delta y+v_{b}\left(A_{n}^{y}-A_{s}^{y}\right) \delta x=0
\end{array}
$$

where the notation is explained in Figure 5.

\section{Discretisation of the momentum equations}

The momentum equations are discretised in a control volume with the velocity as center. In Figure 6 the control volume is drawn for the $x$-momentum equation for an open cell (left) and a cell that is partly cut by the geometry (right). All the terms of the Navier-Stokes equations are discretised in these control volumes using the finite volume method. The discretisations of the different terms are explained in [9].

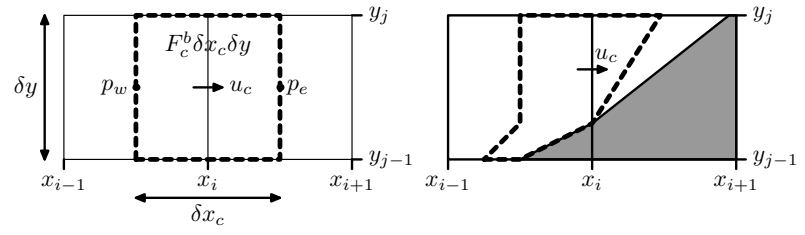

Figure 6. A control volume for the discretisation of the Navier-Stokes equation in $x$-direction in the case of an uncut cell (left) and a cut cell (right).

\section{Temporal discretisation and solution method}

The continuity and Navier-Stokes equations are discretised in time using the forward Euler method. This first order method is accurate enough, because the order of the overall accuracy is already determined by the first order accuracy of the free surface displacement algorithm. Using superscript $n$ for the time level, the temporal discretisation results in

$$
\begin{gathered}
M^{0} u_{h}^{n+1}=-M^{b} u_{b}^{n+1}, \\
\Omega \frac{u_{h}^{n+1}-u_{h}^{n}}{\delta t}=-C\left(u_{h}^{n}, u_{b}\right) u_{h}^{n}-\frac{1}{\rho}\left(\left(M^{0}\right)^{\mathrm{T}} p_{h}^{n+1}-\mu D u_{h}^{n}\right)+F_{h}^{n} .
\end{gathered}
$$

The continuity equation is discretised at the new time level to ensure a divergence free velocity field. The spatial discretisation is written in matrix notation where $M$ is the divergence operator with $M^{0}$ working on the interior velocities and $M^{b}$ on the boundary velocities, $\Omega$ contains cell volumes, $C$ contains the convection coefficients (which depend on the velocity vector) and $D$ contains diffusive coefficients.

To solve the system of equations, the equations are rearranged to

$$
u_{h}^{n+1}=\tilde{u}_{h}^{n}+\delta t \Omega^{-1} \frac{1}{\rho}\left(M^{0}\right)^{\mathrm{T}} p_{h}^{n+1},
$$

where

$$
\tilde{u}_{h}^{n}=u_{h}^{n}-\delta t \Omega^{-1}\left(C\left(u_{h}^{n}\right) u_{h}^{n}-\frac{\mu}{\rho} D u_{h}^{n}-F_{h}^{n}\right) .
$$

First, an auxiliary vector field $\tilde{u}_{h}^{n}$ is calculated using Eq. (10). Next, Eq. (9) is substituted in Eq. (7) which results in a Poisson equation for the pressure. From this equation the pressure is solved using the SOR (Successive Over Relaxation) method where the optimal relaxation parameter is determined during the iterations [1]. Once the pressure field is known, the new velocity field is calculated from $\tilde{u}_{h}^{n}$ using the pressure gradient. 


\section{HANDLING OF THE FREE SURFACE}

After the new velocity field has been calculated, the free surface can be displaced. This is done using an adapted version of the volume-of-fluid method first introduced by [8]. A piecewise constant reconstruction of the free surface is used, where the free surface is displaced by changing the VOF value in a cell using calculated fluxes through cell faces.

The original VOF method has two main drawbacks. The first is that flotsam and jetsam can appear, which are small droplets disconnecting from the free surface [14]. The other drawback is the gain or loss of water due to rounding of the VOF function. By combining the VOF method with a local height function [9], these problems do not appear any more. The local height function is adopted in the following way. For every surface cell, locally a height function is defined, which gives the height of the water in a column of three cells as in Figure 7. The direction in which the function is defined is the direction of the coordinate axis that is most normal to the free surface. Then not the individual fluxes of the three cells are updated, but the height function is updated using fluxes through the boundaries of the column of the three cells (the dashed-lined region in Figure 7). The individual VOF values of the three cells are then calculated from the height of the water in the column. When using this adopted fluiddisplacement algorithm, the method is strictly mass conservative and almost no flotsam and jetsam appear.

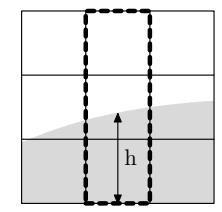

Figure 7. The VOF function in cells near surface cells is updated using a local height function.

To compare the improved VOF method with the original VOF method, a dambreak calculation, where fluid is flowing into an empty domain, has been performed with both methods. In Figure 8 a snapshot is shown of the free surface of the breaking dam flow at the end of the calculation, using standard VOF from Hirt-Nichols. There are a lot of small droplets close to the free surface, which are due to the reconstruction and displacement of the free surface. Also, mass is not conserved in the domain, but about $7 \%$ of the water was lost. The resulting free surface using the VOF method combined with a local height function is shown in the snapshot in Figure 9. The number of droplets is much smaller than in the original VOF-method and mass is perfectly conserved. The remaining droplets, which are partly droplets that are stick to the container walls, only occur at the moment that extreme violent wave impacts occur.

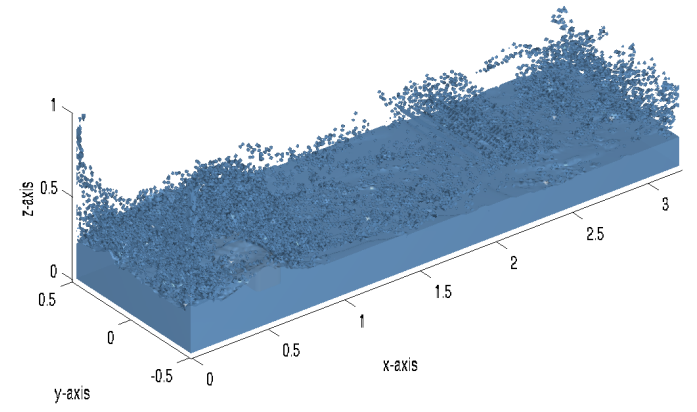

Figure 8. Snapshot at the end of dambreak flow simulation using original VOF

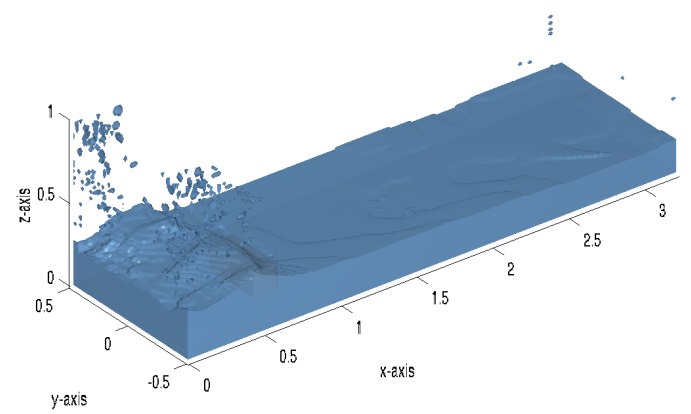

Figure 9. Snapshot at the end of dambreak flow simulation using VOF combined with a local height function

The improved VOF method has also been compared with the commercial code FLOW3D, which also uses a VOF based method for the free surface displacement (see [7] for detailed results). A box has been positioned just above the calm water surface, after which the crest of a high wave hits the box (see Figure 10). In Figure 11 the calculated horizontal force using COMFLOW and FLOW3D are plotted, showing that both codes give qualitatively the same results.

\section{MOVING OBJECTS IN COMFLOW}

In the domain an object, which moves according to a prescribed or calculated motion, can be present. Every time step the object is moved, so new geometry apertures for the cell vol- 


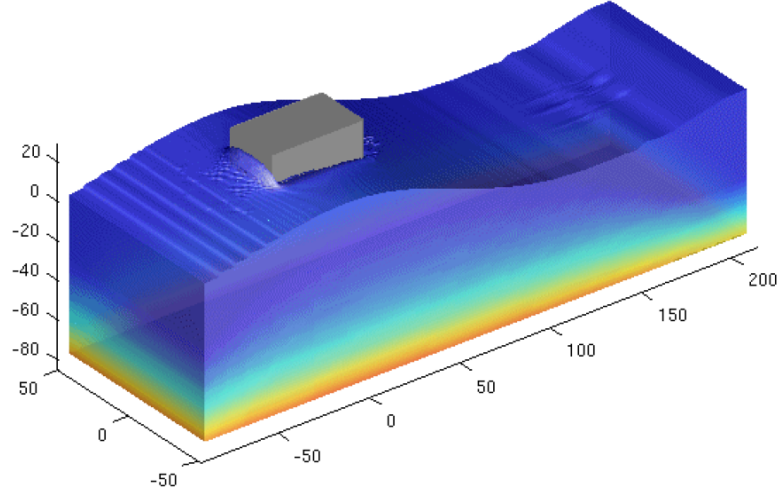

Figure 10. Snapshot of a wave hitting a box positioned just above the calm water surface

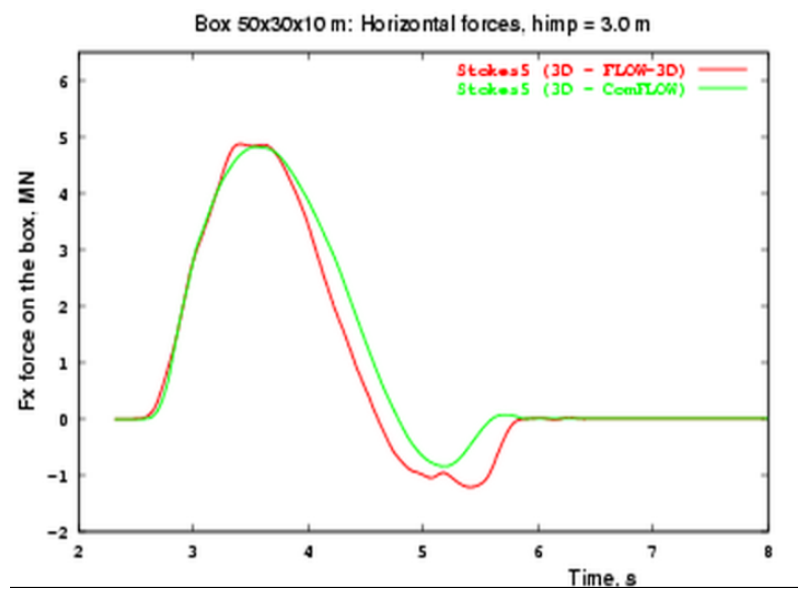

Figure 11. Horizontal force on the box above the water using COMFLOW and the commercial code FLOW3D

umes $F^{b}$ and the cell edges $A^{x}, A^{y}$ have to be calculated. This calculation must be as accurate as possible, because this has a large influence on the smoothness of the pressure field. When the apertures are not calculated exactly, the object seems to be 'breathing' in time, which causes irregularities in the pressure signal.

In two dimensions, the apertures can be calculated almost exactly. In [5] a procedure has been explained how to do this. When using an exact calculation of apertures in three dimensions, cross-sections of polyhedrons with the rectangular grid are needed, which can not be determined in a very straightforward manner. Therefore, in three dimensions a more simple method is adopted, which approximates the three-dimensional body geometry.

The general procedure can be described in three steps. First, the starting geometry is stored in a special way using markers.
Then, every time step the volume apertures are calculated by moving the markers. Finally, the edge apertures are calculated, based on the volume apertures.

At the start of a simulation, the geometry is built from the finite element description given by the user. To calculate the volume and edge apertures, the object is filled with a subgrid of markers. For every cell the number of markers of the cell inside the object is counted, determining the part of the cell that is occupied by an object. If a moving object is present in the domain, the geometry of the object should be stored, such that it can be displaced every time step. Therefore, the markers inside a moving object are stored in an array. Around each marker, a small rectangular volume is defined, such that the union of all the volumes forms the object. To prevent unnecessary storage, the markers in a computational cell that is completely solid will be replaced by one marker with accompanying volume equal to the volume of the computational cell.

During the simulation the volume and edge apertures in the computational grid change every time step. New volume apertures must be calculated with the use of the markers and volumes defined at the start of the simulation. First, the markers are moved according to the motion of the rigid object. In case of a rotation of the object, also the volumes belonging to the marker cells should be rotated. To calculate volume apertures, the cross-sections of the marker volumes with the computational cells should be calculated. For a general rotated volume, this is very complicated in three dimensions. To avoid the calculation of these difficult cross-sections, the marker volumes are not rotated, but are staying grid aligned as in the right of Figure 12. The errors introduced by keeping the volumes grid aligned, namely small holes or small overlapping regions, are not very large.
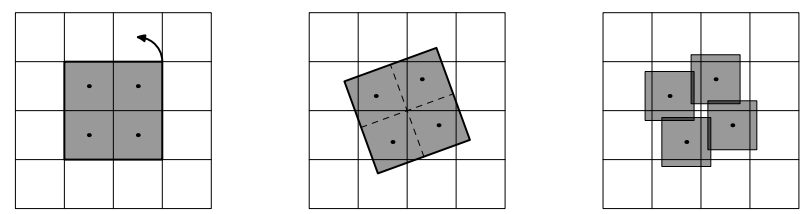

Figure 12. Rotation of a square: starting situation (left); exact rotation (middle); rotation where the marker volumes are kept grid aligned (right).

After the volume apertures have been calculated at the start of every time step, the edge apertures must be determined. The edge apertures are calculated using a piecewise linear reconstruction of the geometry. This method is often used for the reconstruction of the interface between two fluids, as explained in [14]. First, in every cell the normal of the body is calculated based on the filling ratio's of adjacent cells. Using this, a linear approximation of the body geometry in the cell is created where the 
filling ratio of the cell is needed. The edge apertures are determined by the fractions of the cell faces that are cut by the linear approximation. In [5] it has been shown that the edge apertures calculated in this way behave smoothly in time.

\section{COUPLING COMFLOW TO AN OUTER DOMAIN}

In order to initialise simulations of wave loading on floating or fixed structures, COMFLOW has been coupled to a linear diffraction code. A linear code has been selected in order to gain experience on how far this method can be stretched as a first step towards developing an engineering tool. The diffraction code is able to calculate the wave kinematics and vessel motions, but can not calculate the local flow phenomena close to the vessel. These will be calculated by COMFLOW, which is able to produce arbitrary interface configurations and can predict local wave impact. However, the use of ComFLOW is limited to the close surroundings of the vessel because of the required computational effort. So, as sketched in Figure 13, first the diffraction code is used to calculate the far wave field and the vessel motions and then COMFLOW calculates the local wave dynamics. This is a one-way coupling, the RAO's calculated by the diffraction code are used to give the initial flow in the complete COMFLOW domain and to prescribe velocities, pressure and water height at the boundaries of the COMFLOW domain during the time domain simulation.

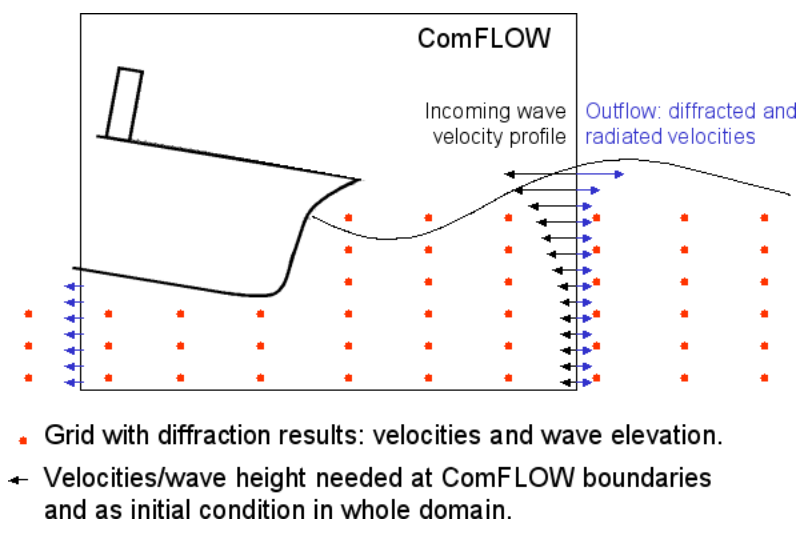

Figure 13. A diffraction code calculates the vessel motions and wave kinematics at a grid of points; these are used to give the initial configuration in the smaller COMFLOW domain and the inflow and outflow velocities at the domain boundaries

An interface has been created, for coupling of the inner COMFLOW to the outer diffraction domain. Output from the interface is the kinematics of the wave field and the ship motion, ready to be used by COMFLOW. The interface consists of the following steps:
1. Read the output of the diffraction analysis program. The diffraction program has been run for a specified geometry at a range of wave frequencies and directions and has produced RAO's (or transfer functions) for 6 DOF vessel motions, and pressures and velocities in a grid surrounding the vessel (the red dots in Figure 13.

2. Specify wave conditions. Several conditions can be chosen, for example conditions based on the JONSWAP spectrum, or a regular wave.

3. Generate time series using the RAO's of the diffraction analysis.

4. Calculate motions of the vessel and select from the time series a critical event by specifying a start and end time for the ComFLOW simulation. This critical event can for example be an event with a steep wave, or an event where the deck level of the ship is exceeded by a high wave.

5. Interpolate the RAO's to the grid used in COMFLOW (which will in general be much finer than the grid on which diffraction results are knows) and write data to a file. The data needed by COMFLOW are the initial velocity and pressure field and initial position of the vessel, and thereafter the boundary conditions at the borders of the domain and vessel motions.

\section{SIMULATION OF AN IRREGULAR WAVE}

The first test case which has been run with the domain decomposition method is the simulation of a two-dimensional linear irregular wave without an object in the flow. The wave has wave height $H_{s}=0.455 \mathrm{~m}$, period $T_{p}=12 \mathrm{~s}$ and the water depth is $400 \mathrm{~m}$. The JONSWAP wave spectrum has been used with 80 frequencies. The COMFLOW domain is $250 \mathrm{~m}$ long. The kinematics calculated by the diffraction code have been prescribed at the inflow and outflow boundary. The kinematics above the mean sea level are calculated using Wheeler stretching, because no theoretically correct linear wave kinematics exist there. The result of COMFLOW perfectly agrees with the predicted linear wave (see the left of Figure 14, where theory and simulation are on top of each other). This demonstrates correct implementation of the interface, the assumption that these waves behave linearly, and that COMFLOW is run with sufficient resolution and does not introduce unphysical nonlinearities for this wave train.

When using the same wave, but ten times higher $\left(H_{S}=4.55\right.$ $\mathrm{m})$, the wave has become nonlinear. There are differences now between COMFLOW and the predicted linear wave (see the right of Figure 14). These differences have two origins. Firstly, the wave has become nonlinear, so the predicted wave using linear theory is not correct any more. Secondly, reflections from the outflow boundary occur, because the velocities prescribed using the linear diffraction code are not correct any more and do not fit to the interior of COMFLOW. To prevent this problem of reflections, another procedure than just prescribing velocities from 

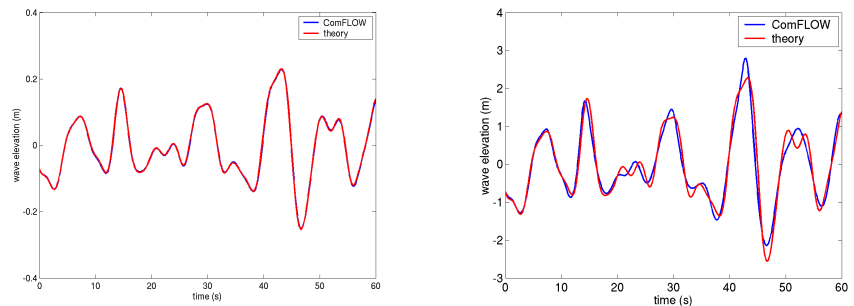

Figure 14. Time trace of an irregular linear wave (left) and nonlinear wave (right) computed with prescribed velocities from linear diffraction theory; the time trace is taken halfway the COMFLOW domain.

the diffraction code should be used on the outflow boundary. For example, a damping zone could be used. But also more sophisticated methods can be implemented, because already a reasonable guess for the velocities is present from the diffraction results. For example, a damping zone which just damps the difference between the COMFLOW velocities and the diffraction velocities could be used. This will be investigated further in a future project.

\section{SIMULATION OF GREEN WATER LOADING}

The goal of the coupling of COMFLOW to a diffraction code is to simulate realistic wave events and calculate the accompanying forces on offshore structures. To test the idea, a simulation of green water loading is performed using the same wave field characteristics as in an experiment performed by Buchner [2]. In the experiment a free floating FPSO has been placed in regular waves with period 12.9 seconds, wave height 13.52 meters and wavelength 260 meters. The total water depth is 150 meters. Measurements were done of the wave in front of the FPSO, relative wave heights in the neighbourhood of the FPSO, water heights and pressures at the deck of the FPSO (see Figure 19) and the pressure at some places in a deck structure. The FPSO has a total length of 260 meter and is 47 meter wide. The draft is 16.5 meter, the total height of the deck at the fore side of the FPSO is 25.6 meter. There is a bulwark extension of 1.4 meter. At the deck, a box-like structure has been placed at which forces and pressures have been measured. The bow has a full elliptical shape without flare.

Two simulations have been run. In the first simulation, the wave and vessel motions are determined by the measurements of the experiment. In [10] an extensive study of the results of this simulation is given. The second simulation is initiated using a diffraction calculation for the far field kinematics and the vessel motions. The diffraction code that is used is a linear diffraction code, developed at MARIN. Although the circumstances are very nonlinear, the linear approximation is thought to be a good start to investigate the possibilities of the method. The results of the domain decomposition method are compared with the exper- iments and also with the first simulation method.

\section{Simulation using the domain decomposition}

First, the diffraction code has been run, from which the vessel motion RAO's and the kinematics at a grid of points around the vessel are written to a file. In Figure 15 the predicted heave and pitch motion of the vessel (heave motion is measured at the center of gravity) is compared with the measured motion. The heave and pitch motion are the most important motions for the prediction of green water on the deck, which occurs when the deck level is exceeded by the water level. As can be seen from the figure, the motions are well predicted by the linear diffraction theory. Only a slight shift in the predicted heave motion is observed and a small reduction of the amplitude.
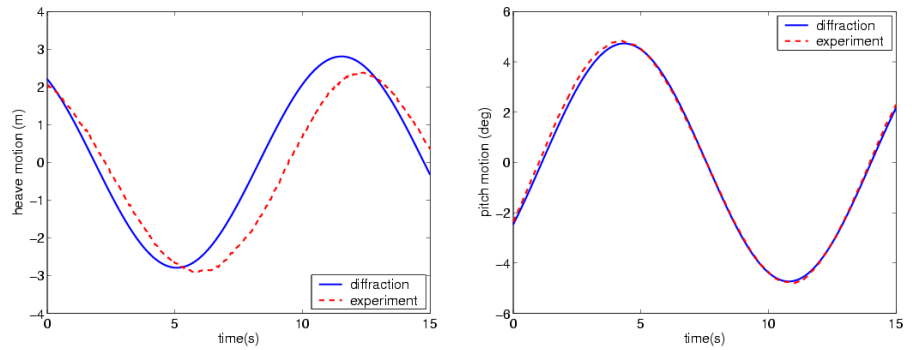

Figure 15. Ship motion predicted by linear diffraction theory and measured during the experiment: heave (left) and pitch (right).

These vessel motions are used to prescribe the motion in the COMFLOW simulation. Furthermore, the initial velocity field and wave height are prescribed using the diffraction results. The grid on which the kinematics are calculated in the diffraction code consists of $61 \times 21 \times 13$ grid points with a distance between the grid points of 13,13 and $12.5 \mathrm{~m}$ in $x, y$ and $z$-direction respectively. The COMFLOW domain is focused on the bow of the ship, and reads about half a wavelength up front and half the ship length aft of the bow. The $y$-coordinate has values between -100 and $100 \mathrm{~m}$, whereas in $z$-direction the domain is cut off at $-100 \mathrm{~m}$. Selecting a grid for such computations is always a compromise between accuracy, computer memory and computing time. At the boundaries of the domain the three velocity components and the water height are prescribed using the results of the diffraction code. A grid of $112 \times 80 \times 76$ grid points is used in the COMFLOW simulation with stretching towards the bow of the ship. Using this grid, cells in the neighbourhood of the bow have sizes in $x, y$ and $z$-direction of $1.4 \mathrm{~m}, 2.0 \mathrm{~m}$ and $1.1 \mathrm{~m}$ respectively. The simulation has been carried out for 15 seconds.

To investigate the behaviour of the wave close to the bow, relative wave probes have been positioned at 30 and 5 meters in front of the bow. In Figure 16, the relative wave height calculated 
by COMFLOW is compared with the experiment. The agreement between measurement and calculation is good, indicating that the combined vessel motion and wave is well predicted.
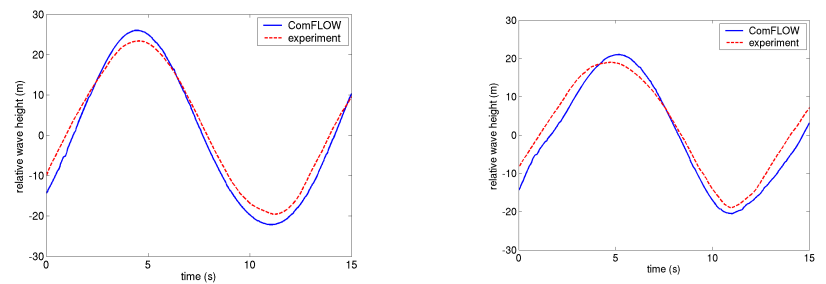

Figure 16. Relative wave height 30 meters (left) and 5 meters (right) in front of the bow of the FPSO.

Figure 17 shows contours describing the propagation of the water front on the deck of the FPSO. Buchner [2] describes the water flow on the deck in the following words: First, the horizontal velocity of the water front on the deck is almost zero. Then, the water front starts to translate onto the deck with a similar velocity from all sides, perpendicular to the local deck contour. Finally, the water contours from the front and sides meet at the centerline of the ship and result in a high velocity 'jet', which flows with a high velocity aft along the middle of the deck. These stages can be recognised in the contour plots in Fig 17. In the experiment, the time interval between two contours is $0.31 \mathrm{~s}$, and in the simulation the difference is $0.30 \mathrm{~s}$. The agreement between the propagation of the water front in experiment and simulation is rather good. The water jet is formed a bit earlier in the simulation than in the experiment.

In Fig 18 a snapshot of the simulation at time $7.5 \mathrm{~s}$ is shown, where the high velocity jet is very well visible.
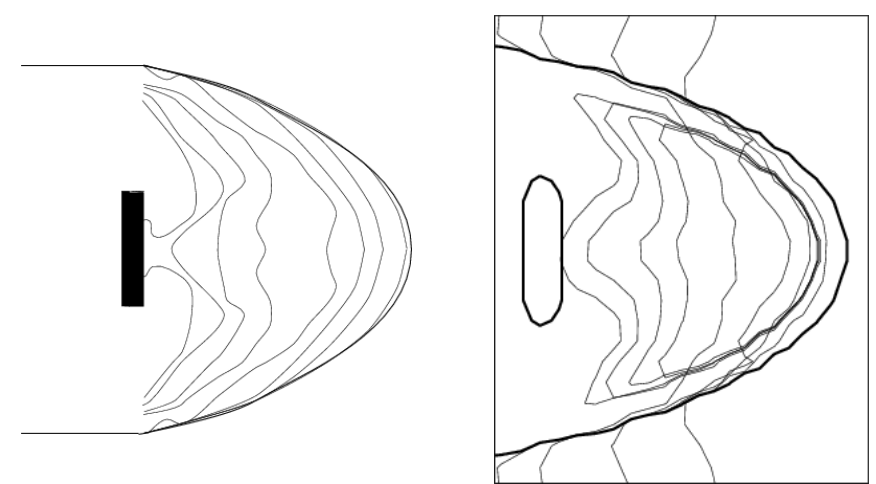

Figure 17. Contours of the water front propagating over the deck of the FPSO, model test every $0.31 \mathrm{~s}$ (left) and COMFLOW every 0.30 $S$ (right).

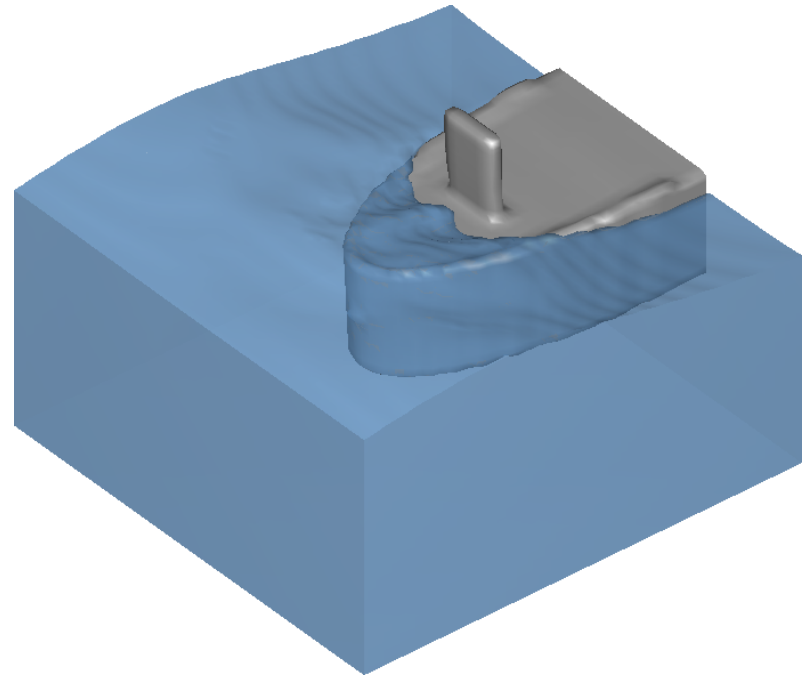

Figure 18. Snapshot of a simulation with green water on the bow of the FPSO.

To make a further comparison of the behaviour of the water on the deck with the experiment, the measurements of the water probes at the deck and the pressure panels at the deck have been used. Figure 19 shows the measurement positions of the water probes and pressure panels at the deck of the FPSO: at four positions the water height is measured, with distance between two of them of $10 \mathrm{~m}$; the distance between the pressure panels that are positioned in between the water height probes is also 10 meters, with $\mathrm{P} 1$ positioned $7 \mathrm{~m}$ left of $\mathrm{H} 1$. Height probe $\mathrm{H} 4$ is positioned just in front of the deck structure.

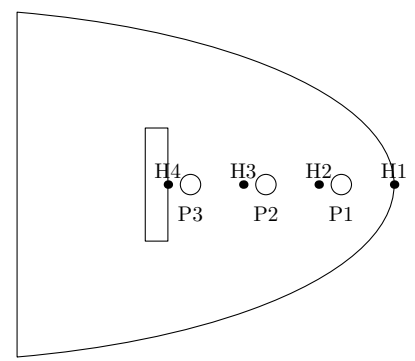

Figure 19. Positions of measured pressure and water height at the deck of the FPSO.

In Figure 20 the water height at the deck at positions $\mathrm{H} 1$ and $\mathrm{H} 3$ is shown. The moment the water reaches the water probes is very well predicted by COMFLOW. The amount of water on the deck is larger than in the experiment. Close to the deck edge (at H1) the water height is 2 meter higher than measured in the 
experiment and further on the deck at $\mathrm{H} 3$, it is still 1.5 meter too high; note that this difference is less than two grid cells. The second hump in the left of Figure 20 is predicted by COMFLOW at the same moment as in the experiment. This hump is present due to the water returning from the deck structure. In Figure 21, the pressure at the deck at positions $\mathrm{P} 1$ and $\mathrm{P} 3$ is shown. The same conclusion can be drawn from this plot as from the plotted water heights: the amount of water on the deck is too large, but further aft on the deck the agreement becomes better. In both pictures of Figure 21 an oscillating behaviour of the pressure can be observed. Every oscillation represents a switch of the monitoring point fixed at a moving structure to another cell. The pressure value changes when such a switch of cell happens, because the pressure is positioned in the cell center.
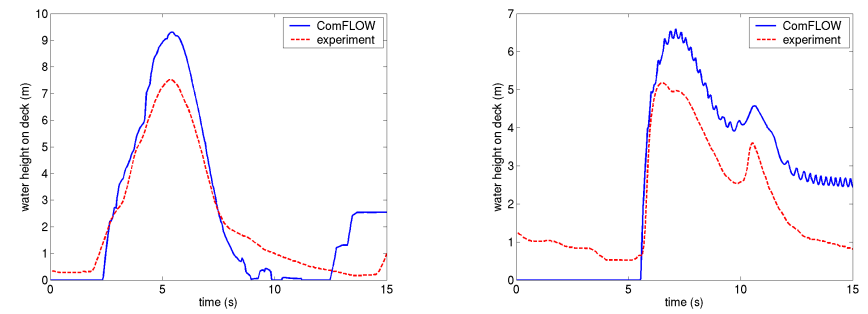

Figure 20. Water height at the deck close to the bow H1 (left) and closer to the deck structure $\mathrm{H} 3$ (right).
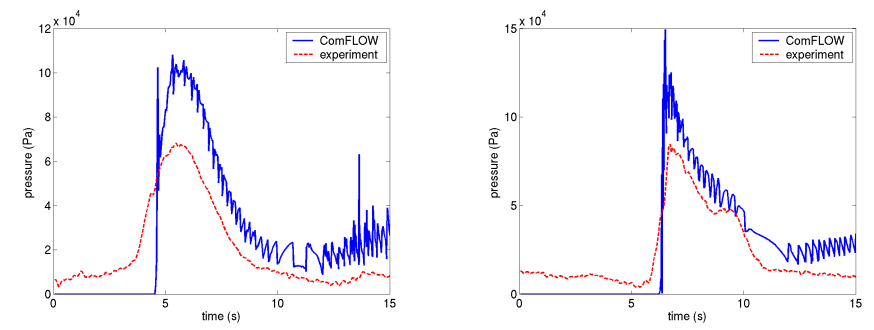

Figure 21. Pressure at the deck close to the bow P1 (left) and close to the deck structure P3 (right).

At the deck structure also pressure panels are positioned to measure the wave impact. The lowest panel is positioned $2.4 \mathrm{~m}$ above the deck level. The time trace of the load on that panel is shown in Figure 22, which shows a good agreement between simulation and experiment. The peak of first impact is too high in the simulation, but it is not clear whether this peak is physical or whether it is originating from the numerical calculation.

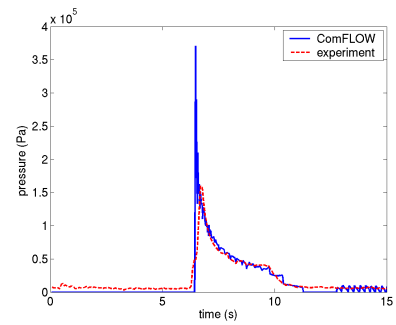

Figure 22. Pressure at the center line of the deck structure, $2.4 \mathrm{~m}$ above the deck.

\section{Comparison with simulation initialised using experi- mental data}

As described above, two simulation methods for green water loading are used. In the previous section a domain decomposition of COMFLOW and a far field using a diffraction code is presented. In this section the results are compared with the results of COMFLOW initiated using the experiment. In this simulation the motion of the FPSO is prescribed from the measurements of the experiment. The wave is generated at the inflow boundary by a superposition of linear components. The components are deduced from the time signal of a wave probe at $230 \mathrm{~m}$ in front of the bow. The side walls are solid walls and at the outflow boundary a non-reflecting condition is used. The upper part of Figure 23 shows the pressure and water height at the deck (positions P1 and H3, see Figure 19). The results of the two simulations have been compared with the measurements. At the forward part of the deck both simulation methods predict too much water as can be seen from the pressure at P1. Further aft on the deck the water height is better predicted by the simulation method using domain decomposition. The water height calculated using the measurements to initiate COMFLOW is too low. This results in smaller loads on the deck structure as can be seen from the bottom of Figure 23. There the pressure at the deck structure, $2.4 \mathrm{~m}$ above the deck level, is plotted. The picture is zoomed in to be able to compare the results of the two methods with the experiment. Concluding, using the domain decomposition method gives superior results above initiating COMFLOW with the experimental data.

\section{Grid refinement}

The simulation of green water on the deck has been run on three different grids to investigate the behaviour under grid refinement. The grid of points on which the diffraction results are calculated is not changed, only the COMFLOW grid is altered. The number of grid points used is $56 \times 40 \times 38,84 \times 60 \times 57$, and $112 \times 80 \times 76$. All three grids are focused towards the bow of the FPSO.

Figure 24 shows the water height at position $\mathrm{H} 2$ at the deck 

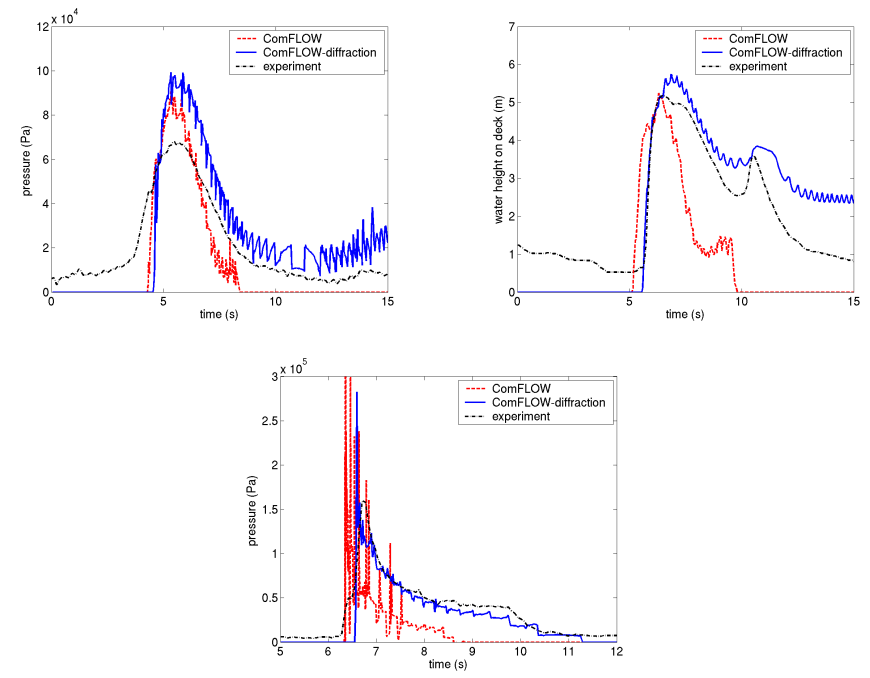

Figure 23. Pressure at position $\mathrm{P} 1$ (left), water height at position $\mathrm{H} 3$ (right) and pressure at deck structure (bottom): COMFLOW driven by experimental results and by the diffraction code compared.

of the ship (see Figure 19) and the pressure at P3. The results on the finest grid are clearly better than the coarse grid results. At position $\mathrm{H} 2$, where the water height on the deck is measured and calculated, the difference between the results on the three grids is not very large. Only at the end, the coarsest grid gives a raise in the water height that is not seen at the other grids or in the experiment. Closer to the deck structure, at P3, the difference between the grids is larger. The coarsest grid is clearly not good enough, whereas the finest grid gives the best results.
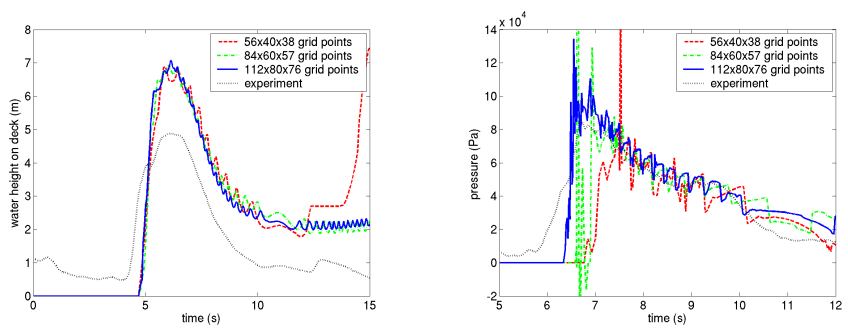

Figure 24. Water height $\mathrm{H} 2$ and pressure $\mathrm{P} 3$ at the deck of the FPSO calculated on three different grids.

\section{CONCLUSIONS}

In this paper a method to simulate wave loading resulting in green water on the deck of a floating FPSO has been described. For the flow localized at the bow of the vessel, a Navier-Stokes solver COMFLOW is used with a Volume-of-Fluid based free surface displacement. For the far field, a linear diffraction code is used, which calculates the fluid kinematics at a grid of points and the ship motion. These are used to initialize COMFLOW and to prescribe ship motion and fluid kinematics at the boundaries during the simulation.

An interface has been developed, which reads the data of the diffraction code and gives as output the initial wave field in ComFLOW, the ship motions and the wave kinematics at the domain boundaries. Wave conditions can be selected. By examining the time traces of waves and ship motions, extreme events can be chosen which will likely give green water at the ship's bow. Then, this event can be simulated in the COMFLOW domain which is focussed around the bow of the ship.

As a start, an irregular wave has been simulated using the far field wave kinematics produced by the linear diffraction code. When the wave amplitude is small (such that a linear wave is formed) the results of COMFLOW are almost exactly the same as the linear diffraction code predicts. But for higher amplitude waves, the COMFLOW results differ from the predicted wave by the diffraction code, because the nonlinearities of the wave are developing within the COMFLOW domain.

Next, results of a green water simulation have been shown and compared with model test results. The ship motions predicted by the linear diffraction code are pretty much the same as in the experiment. When using the prediction of ship motions and wave kinematics in COMFLOW, the results for the flow on the deck show a reasonable agreement with the experiment. The amount of green water on the deck is somewhat larger than in the model test. The pressure load at the deck structure compares very well. The results of the domain decomposition method have also been compared with a simulation of COMFLOW where the wave field and ship motion are prescribed using the experimental results. The former simulation shows better results, especially close to the deck structure.

Concluding, the method of decomposing the domain in a far field and a domain with the close surroundings of the ship gives already good results when using a linear diffraction code for the far field. Using a domain decomposition method is a very promising way to simulate wave loading and green water, because the ship motions and wave kinematics on the boundaries of the COMFLOW domain are calculated by a cheaper method. The relatively expensive COMFLOW method only has to be used for the close surroundings of the vessel. The next step in this investigation will be to use a nonlinear code to calculate the far field kinematics and ship motions and investigate the consequences on the results. Another possible step is to calculate ship motions within the program by using integrated pressures as exciting forces. This has already been investigated for a falling wedge into initially calm water $[5,9]$. 


\section{ACKNOWLEDGMENT}

The SAFE-FLOW project (SAFE-FLOating offshore structures under impact loading of shipped green water and Waves) is funded by the European Community under the 'Competitive and Sustainable Growth' Programme (EU Project No.: GRD1-200025656) and a group of 26 industrial participants (oil companies, shipyards, engineering companies, regulating bodies). The authors are solely responsible for the present paper and it does not represent the opinion of the European Community.

\section{REFERENCES}

[1] Botta, E., and Ellenbroek, M., 1985. "A modified SOR method for the Poisson equation in unsteady free-surface flow calculations". J. Comput. Phys., 60 , pp. 119-134.

[2] Buchner, B., 2002. Green water on ship-type offshore structures. PhD Thesis, University of Delft, Delft, The Netherlands, November.

[3] Daalen, E., Kleefsman, K., Gerrits, J., Luth, H., and Veldman, A., 2001. "Anti-roll tank simulation with a volume of fluid (vof) based navier-stokes solver". In Proceedings of 23rd Symp. on Naval Hydrodynamics, Val de Rueil, France. pp. 457-473.

[4] Fekken, G., Veldman, A., and Buchner, B., 1999. "Simulation of green-water loading using the navier-stokes equations". In Proceedings 7th Intern. Conf. on Numerical Ship Hydrodynamics, Nantes.

[5] Fekken, G., 2004. Numerical simulation of free-surface flow with moving objects. $\mathrm{PhD}$ Thesis, University of Groningen, Groningen, The Netherlands, March. See also URL http://www.ub.rug.nl/eldoc/dis/science/ g. fekken.

[6] Gerrits, J., and Veldman, A., 2003. "Dynamics of liquidfilled spacecraft”. J. Engrg. Math., 45, pp. 21-38.

[7] Iwanowski, B., 2004. Benchmarks of the comflow program. Tech. Rep. TR-2003-0064, SafeFLOW JIP.

[8] Hirt, C., and Nichols, B., 1981. "Volume of fluid (VOF) method for the dynamics of free boundaries". J. Comput. Phys., 39 , pp. 201-225.

[9] Kleefsman, K., Fekken, G., Veldman, A., Iwanowski, B., and Buchner, B. "A Volume of Fluid based simulation method for wave impact problems". J. Comput. Phys. Accepted for publication.

[10] Kleefsman, K., and Veldman, A., 2004. "An improved Volume of Fluid iVOF method for wave impact type problems". In Proceedings of OMAE-FPSO 2004, ASME Conferences. Paper Number 0066.

[11] Loots, G., Hillen, B., and Veldman, A., 2003. "The role of hemodynamics in the development of the outflow tract of the heart". J. Engrg. Math., 45 , pp. 91-104.
[12] Mizoguchi, S., 1988. "Analysis of shipping water with the experiments and the numerical calculations". JSNA, 163 .

[13] Mizoguchi, S., 1989. "Design of freeboard height with the numerical simulation on the shipping water". In PRADS' 89.

[14] Rider, W., and Kothe, D., 1998. "Reconstructing volume tracking”. J. Comput. Phys., 141 , pp. 112-152.

[15] Stansberg, C., Hellan, O., Hoff, J., and Moe, V., 2002. "Green sea and water impact: numerical predictions validated against model tests". In Proceedings of OMAE 2002, Oslo, ASME Conferences. Paper Number OMAE200228562.

[16] Zhou, Z., de Kat, J., and Buchner, B., 1999. "A non-linear 3-D approach to simulate green water on deck". In Proceedings 7th Intern. Conf. on Numerical Ship Hydrodynamics, Nantes, France. 